\title{
Measurement of beam energy spread in a space-charge dominated electron beam
}

\author{
Y. Cui, Y. Zou, M. Reiser, R. A. Kishek, I. Haber, S. Bernal, and P. G. O’Shea \\ Institute for Research in Electronics and Applied Physics, University of Maryland, College Park, Maryland 20742, USA
}

(Received 26 May 2004; published 20 July 2004)

\begin{abstract}
Characterization of beam energy spread in a space-charge dominated beam is very important to understanding the physics of intense beams. It is believed that coupling between the transverse and longitudinal directions via Coulomb collisions will cause an increase of the beam longitudinal energy spread. At the University of Maryland, experiments have been carried out to study the energy evolution in such intense beams with a high-resolution retarding field energy analyzer. The temporal beam energy profile along the beam pulse has been characterized at the distance of $25 \mathrm{~cm}$ from the anode of a gridded thermionic electron gun. The mean energy of the pulsed beams including the head and tail is reported here. The measured rms energy spread is in good agreement with the predictions of the intrabeam scattering theory. As an application of the beam energy measurement, the input impedance between the cathode and the grid due to beam loading can be calculated and the impedance number is found to be a constant in the operation region of the gun.
\end{abstract}

DOI: 10.1103/PhysRevSTAB.7.072801

PACS numbers: 29.30.Dn, 29.27.Fh, 29.17.+w, 84.37.+q

\section{INTRODUCTION}

Understanding the physics of the beam energy spread in high-quality intense beams is very important in the applications of advanced particle accelerators for heavyion inertial fusion, high-energy colliders, and freeelectron lasers. If the energy spread or the longitudinal temperature of the beam is too big, the chromatic aberrations in the beam optics will degrade the beam quality and cause difficulty in beam handling. One of the mechanisms causing energy spread growth is the energy transfer from the transverse direction into the longitudinal direction via Coulomb collisions or other collective spacecharge effects. This happens in a system with temperature anisotropy, such as a beam accelerated in the longitudinal direction. In such a system, the longitudinal temperature of the beam is decreased during acceleration, while the temperature in the transverse direction is kept roughly the same as in the cathode. The multiple soft Coulomb collisions and instabilities will try to equilibrate this anisotropic state, causing an increase in the longitudinal beam energy spread or temperature. Many theoretical studies have been made on the description of this energy equipartitioning due to small angle Coulomb collisions [1-3]. However, there are only a few experimental results on this topic. The first experimental observation of enhanced energy spread growth in a low current electron beam (beam current is on the order of $\mu \mathrm{A}$ ) was reported by Boersch in 1954 [4] and since then this phenomenon has been known as the Boersch effect. This energy spread broadening is believed to be due to Coulomb collisions happening at the beam waist. Hyatt, in 1987, reported an experimental measurement of the anisotropic temperature relaxation in a stationary, magnetically confined electron plasma, where he found a good agreement between the experimental results and the small-momentumtransfer collision theory [5]. The energy equipartitioning due to the Coulomb collisions is a relatively long relaxation process. At a distance close to the source, the energy spread due to the longitudinal-longitudinal relaxation [6] is comparable to that due to the Boersch effect.

In this paper we report an experiment conducted at the University of Maryland Electron Ring (UMER) [7] laboratory to study the energy spread growth in a spacecharge dominated beam. The initial energy spread of the beams from a thermionic electron gun has been measured with a high-resolution energy analyzer. The experimental results are in very good agreement with the theoretical lower limit imposed by Coulomb scattering (Boersch effect) and the longitudinal-longitudinal effect. In the following sections, we first describe the experimental setup, which includes a high-resolution energy analyzer. Then we will give the measurement results of beam energy profile and compare them with theoretical predictions. Last, we introduce a method, as a novel application of the energy analyzer, to measure the beam impedance of the cathode inside the electron gun.

\section{HIGH RESOLUTION ENERGY ANALYZER AND EXPERIMENTAL SETUP}

To study and measure the beam longitudinal energy spread in a space-charge dominated beam, several generations of retarding field energy analyzers have been developed at the University of Maryland. Early experiments were carried out with a simple parallel-plate energy analyzer [8] and a cylindrical energy analyzer [9]. Based on the previous design, we developed a retarding field energy analyzer with variable focusing. This energy analyzer achieves a greater resolution of beam energy spread measurements compared with the previous two designs [10]. The resolution of this energy analyzer is $0.2 \mathrm{eV}$ for a $5 \mathrm{keV}$ beam. Figure 1 shows the schematic of the energy analyzer. The electron beam comes from the 


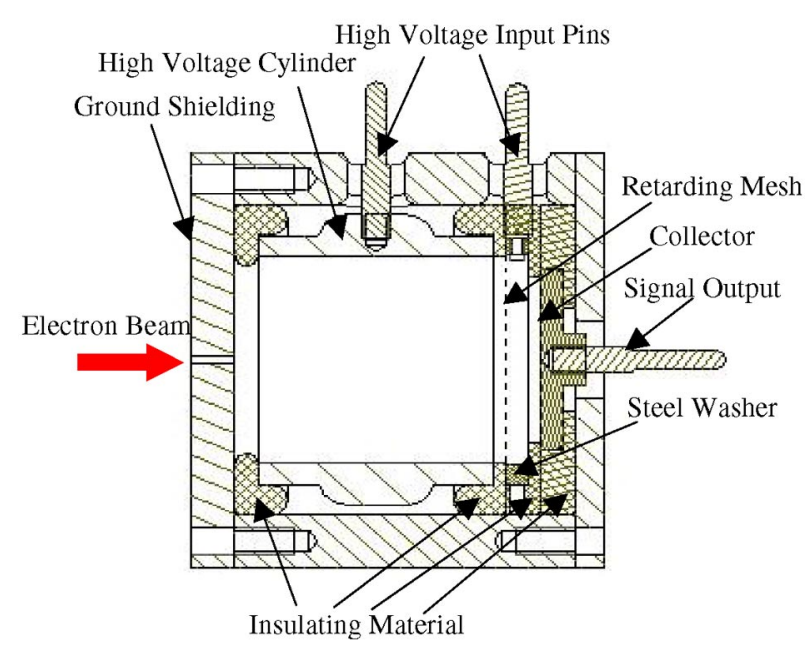

FIG. 1. (Color) Schematic of the energy analyzer with variablefocusing cylindrical electrode. The length is $4.8 \mathrm{~cm}$ and the diameter is $5.1 \mathrm{~cm}$. The aperture size is $1 \mathrm{~mm}$ in diameter.

left. The beam first sees a grounded steel plate with a $1 \mathrm{~mm}$ diameter circular aperture through which a small beamlet passes into the high potential region. The high voltage steel cylinder with a length of $2.5 \mathrm{~cm}$ and an inner diameter of $2.5 \mathrm{~cm}$ is supported by two machinable ceramic (MACOR) rings and is connected to the external high voltage source to provide both deceleration and radial focusing of the beamlet. The retarding grid is a molybdenum wire mesh. The wire diameter is $0.05 \mathrm{~mm}$ and the mesh consists of $20 \times 20$ wires per square centimeter. The transmission rate of the grid, which is defined as the ratio of the open area of the hole to the total area of the surface containing the grid, is $80 \%$. The mesh is soldered to a steel ring with a thickness of $2.5 \mathrm{~mm}$, which is held in place by a MACOR ring with a thickness of $2 \mathrm{~mm}$ and connected to the external high voltage source to provide a retarding and focusing voltage. Behind the high voltage mesh is a copper collector plate, from which the current signal is picked up directly by an oscilloscope. With a length of $4.8 \mathrm{~cm}$ and a diameter of $5.1 \mathrm{~cm}$, this energy analyzer can be easily inserted at any place in the beam line. Figure 2 shows the electrical circuit of the analyzer. The focusing voltage is provided by a battery which is in series with the retarding voltage. From both the experiment and simulation, we found when the focusing voltage is $120 \mathrm{~V}$ higher in amplitude than the retarding voltage, proper focusing will be achieved for a $5 \mathrm{keV}$ beam [10]. When the retarding voltage is at about the same potential as the beam energy, the kinetic energy of the electrons in the beam is too small (almost zero) to generate any secondary emission on the mesh. After electrons pass through the mesh, they will be accelerated to the collector forming a current signal. Possible secondary electrons from the collector will be suppressed by the reverse field on the collector surface, so secondary electrons are not a concern in the device.

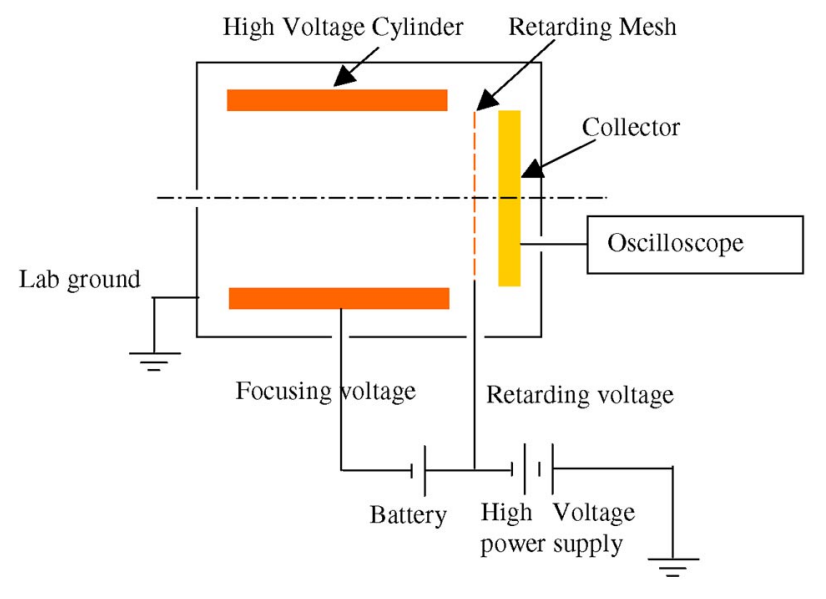

FIG. 2. (Color) The electrical circuit of the energy analyzer with variable-focusing cylindrical electrode.

The experimental setup as shown in Fig. 3 consists of a gridded thermionic electron gun, a Bergoz coil, a solenoidal magnetic lens, and a diagnostic chamber. The Bergoz coil is a high resolution wideband current monitor with a $0.2 \mathrm{~ns}$ rise time. The energy analyzer is located in the diagnostic chamber after the solenoid. The solenoid is used to control the beam current into the energy analyzer. The distances of the solenoid and energy analyzer from the gun are 11 and $24 \mathrm{~cm}$, respectively. The magnetic fringe field extends less than $10 \mathrm{~cm}$ from the solenoid center, so the magnetic field has no influence inside the energy analyzer or electron gun. In the ground shielding of the energy analyzer, there is a $1 \mathrm{~mm}$ diameter pinhole at the front for beam entry. The energy analyzer can be aligned by a linear feedthrough containing three connectors for the retarding voltage, focusing voltage, and output signal. We also developed a computer-controlled system for automating the measurement process. By automatically controlling the retarding voltage and oscilloscope this system can take the energy analyzer data with a very fine step size. The smallest possible change in the retarding voltage is $0.16 \mathrm{~V}$, on top of several kilovolts. We

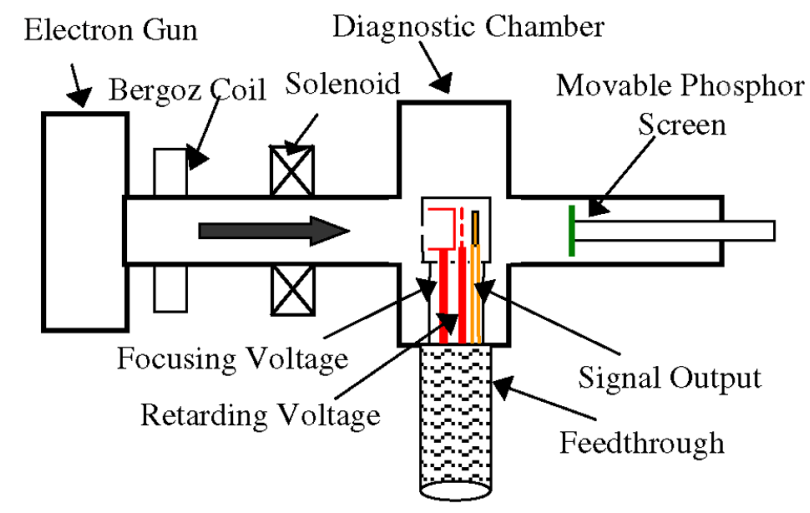

FIG. 3. (Color) The experimental setup. 
also have the ability to insert a movable phosphor screen into the plane of the energy analyzer so that we may obtain an image of the beam at that axial position.

\section{EXPERIMENTAL RESULTS AND ANALYSIS}

In the experiment, the nominal beam energy is $5 \mathrm{keV}$ and the beam current is $135 \mathrm{~mA}$. The beam pulse width is $100 \mathrm{~ns}$ with a rise time of $2 \mathrm{~ns}$, as measured by Bergoz coil, as shown in Fig. 4. The initial beam size and its derivative at the anode of the electron gun are $r_{i}=5 \mathrm{~mm}$ and $r_{i}^{\prime}=0.03 \mathrm{rad}$, respectively. The normalized effective emittance $\varepsilon_{n}$ is $10 \mu \mathrm{m}$. Figure 5 shows the beam envelope from the electron gun to the energy analyzer for three different focusing strengths of the solenoid. For a focusing strength of $104 \mathrm{G}$, the beam envelope is shown with a solid blue line and the blue dots are the beam size from experimental measurement. At this focusing strength, the energy analyzer can get the maximum signal because it is at the beam waist. Figure 6 is a typical output pulse signal from the energy analyzer when the energy analyzer is at the position of the beam waist. The signal amplitude is about $120 \mathrm{mV}$ with a rise time of $5 \mathrm{~ns}$ and the noise is about $\pm 1 \mathrm{mV}$. Noise effects can be reduced after averaging samples. From Fig. 6 we can see that this energy analyzer has a good response, and records the beam signal faithfully compared with the beam current signal from Fig. 4. But in the experiment, in order to reduce the longitudinal space-charge effect inside the device, we need to limit the signal magnitude to about $10 \mathrm{mV}$ [11]. We can control the solenoid focusing strength, and therefore the position of the beam waist, to let the lower current beamlet enter the energy analyzer. In Fig. 5, the black solid line is for the beam envelope with a weak focusing of $85 \mathrm{G}$ and the red line is for a strong focusing of $120 \mathrm{G}$ (the red crosses are the beam size measured from experiment).

During the experiment, the focusing voltage inside the energy analyzer is set to $120 \mathrm{~V}$ relative to the retarding

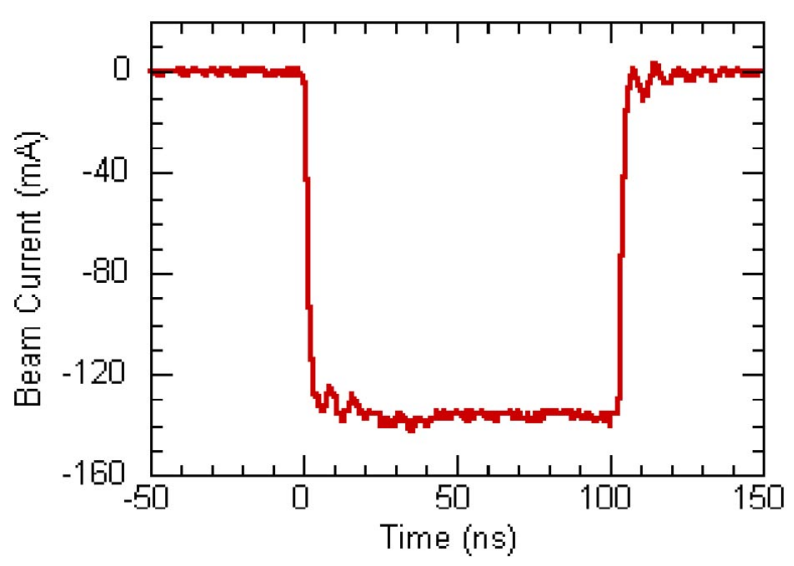

FIG. 4. (Color) Beam current signal measured by Bergoz coil.

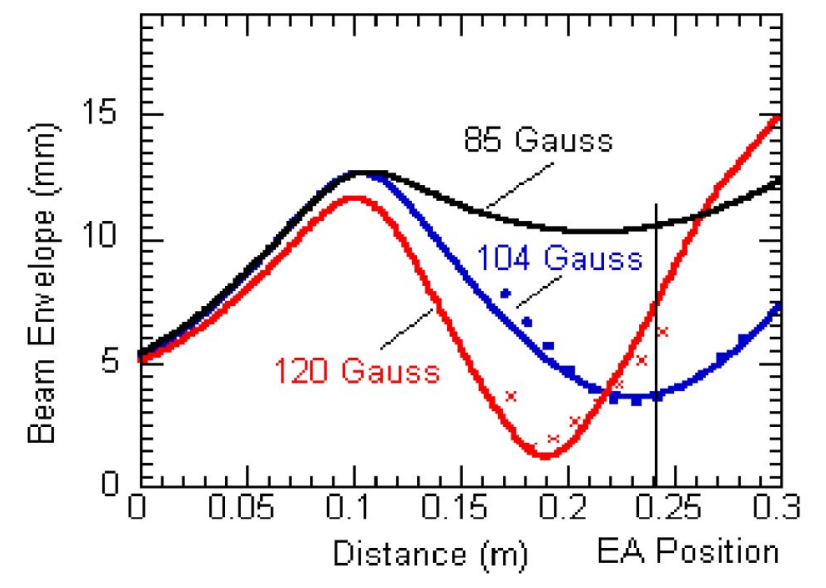

FIG. 5. (Color) Beam envelope from the electron gun to the energy analyzer for three different focusing strengths of the solenoid.

voltage to optimize the device resolution. The retarding voltage is changed by a step size of $0.5 \mathrm{~V}$, which is adequate for the experiment. For a given retarding voltage, 16 current pulses are sampled and averaged to improve the signal-to-noise ratio. In Fig. 7, different signal profiles corresponding to different retarding voltages are plotted together when a weak focusing strength is used (the value of the retarding voltage is referenced to the lab ground). In the figure, we can see that the collector signal decreases with increasing retarding voltage. There are two things in the figure worth noting. First, we can see that the signal at the beam head tends to be larger than in the rest of the beam. This is either due to high-energy particles in the head or the time-varying space-charge effect in the device. Second, there are oscillations in the waveform that increase as the retarding voltage increases. This might be due to a virtual cathode oscillation inside the energy analyzer. The exact mechanisms of these

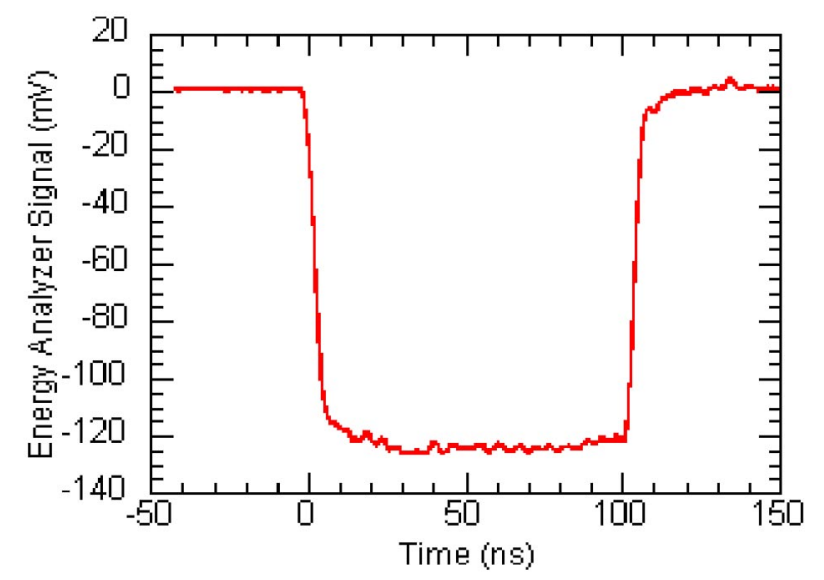

FIG. 6. (Color) A typical output pulse signal from the energy analyzer when the energy analyzer is at the position of the beam waist. 


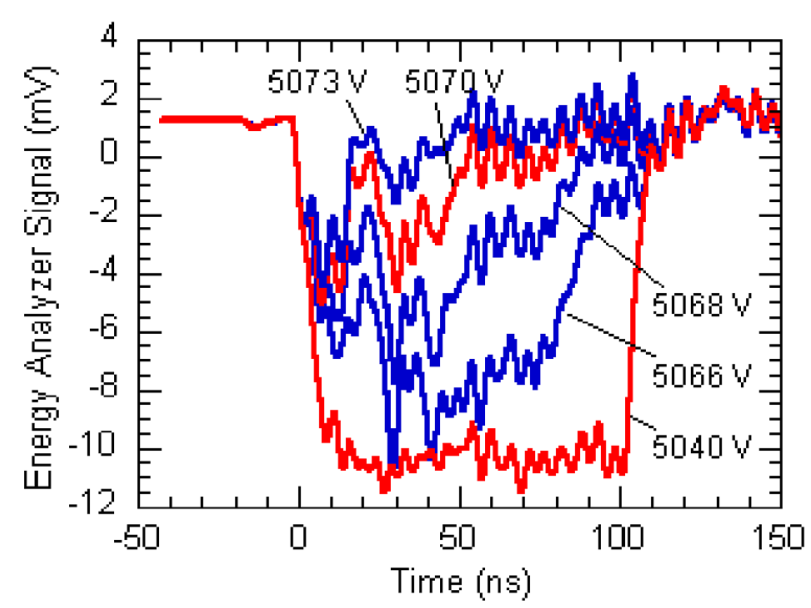

FIG. 7. (Color) Energy analyzer outputs at different retarding voltages.

transient behaviors (within a couple of nanoseconds) inside the device are not well understood and need to be studied in more detail in the future. At this stage, we are focusing on the beam energy measurement at longer temporal scale $(\sim 10 \mathrm{~ns})$.

By differentiating the energy analyzer output with respect to the retarding voltage, we can get the beam energy profile for the whole beam. Figure 8 shows such an energy spectrum with rms energy spread of $2.2 \mathrm{eV}$, FWHM of $3.9 \mathrm{eV}$, mean energy of $5070.5 \mathrm{eV}$, and spectrum peak of $5069.7 \mathrm{eV}$. The sampling point is taken at the middle of the beam pulse.

As we said, the energy spectrum is time resolved. Figure 9 shows the measured mean energy as a function of time along the beam pulse. The measured mean energy of the main beam is about $5070 \mathrm{eV}$. The absolute value of the measured energy is about $50 \mathrm{eV}$ higher than the assumed beam energy from the gun. It is believed that this dc energy shift is due to field leakage through the

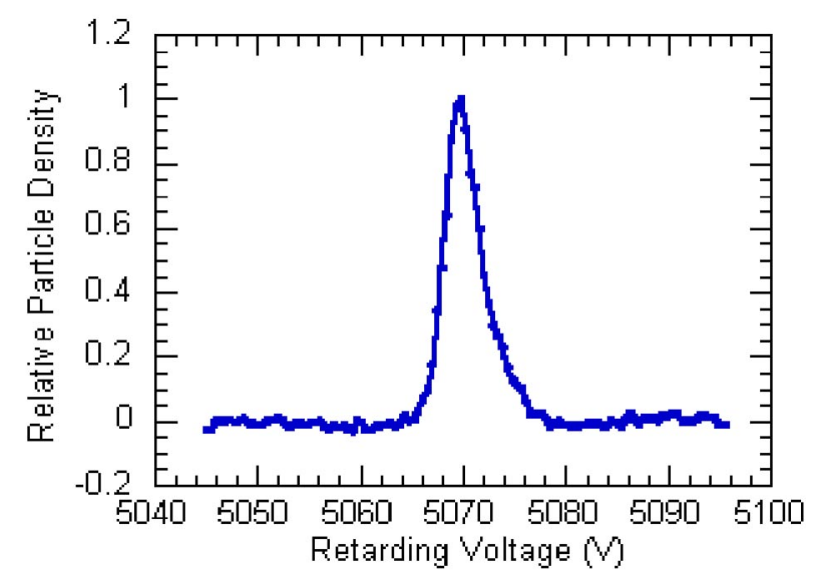

FIG. 8. (Color) Beam energy spectrum for a beam with energy of $5 \mathrm{keV}$ and current of $135 \mathrm{~mA}$. The rms energy spread is $2.2 \mathrm{eV}$.

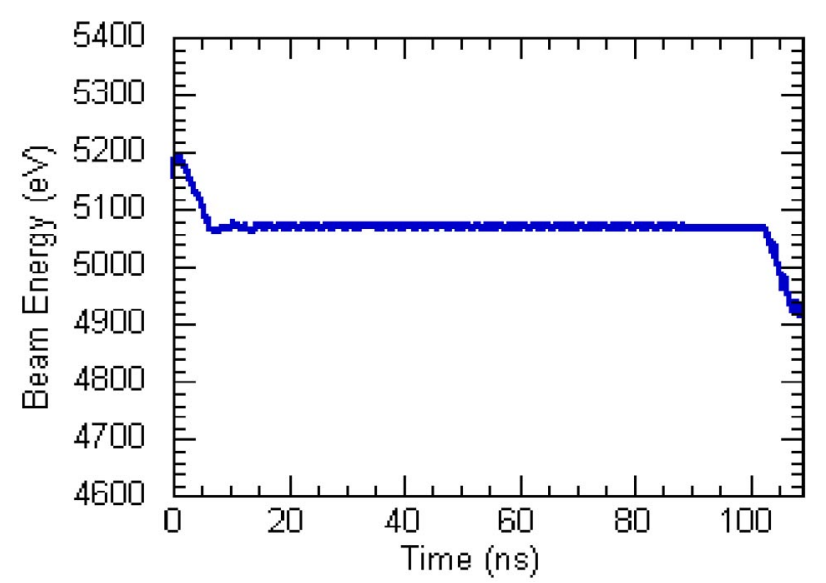

FIG. 9. (Color) Mean energy along the beam pulse for a beam with energy of $5 \mathrm{keV}$.

retarding mesh inside the energy analyzer. From the figure, we can see that the head of the beam has a higher mean energy, up to $5200 \mathrm{eV}$, and the tail of the beam has a lower energy, down to $4940 \mathrm{eV}$. The length of the head and tail is about $5 \mathrm{~ns}$ each. This energy difference at the head and tail is due to the unbalanced collective space charge force. Beam edge expansion of an initially rectangular bunch beam has been studied in Refs. [12-14]. According to the calculation, for a $5 \mathrm{keV}, 135 \mathrm{~mA}$ beam with average radius $7 \mathrm{~mm}$, when the beam is $25 \mathrm{~cm}$ away from the cathode, the front edge is $\sim 1500 \mathrm{eV}$ higher than the beam energy with a rise time of $\sim 1 \mathrm{~ns}$ and the rear edge is $\sim 1500 \mathrm{eV}$ lower than the beam energy with the same drop time for an ideal rectangular pulse employed on the cathode. The theoretical calculation is based on an ideal rectangular beam, but actual beams have finite rise time. In our experiment, the rise time of the pulse signal employed on the cathode in the gridded gun is measured to be around $\sim 2 \mathrm{~ns}$. The system bandwidth of the measurement instruments such as the energy analyzer and the scope also impose a limit on the measurable rise time. In this measurement, we believe the $5 \mathrm{~ns}$ length of the measured beam head/tail is mainly due to the limited system bandwidth. Although the rise time and drop time caused by beam expansion is shadowed by the limited system bandwidth, the energy differences still can be seen in the rise and drop time. It is noticed that the measured beam energy at the head is $130 \mathrm{eV}$ higher than the main beam, while the theory predicts about $1500 \mathrm{eV}$ higher. The difference, we believe, is due to two reasons. The first reason is that the theory assumes a rectangular longitudinal profile with zero rise time; if the rise time has a finite value, the energy gained at the beam head will be lower. Second, the number of particles which have the highest energy is very small in the beam head. It is difficult in experiment to capture these particles due to the limited signal resolution in the device. Nevertheless, it is the first time that we clearly observed the temporal 
mean energy information including the head and tail along the beam pulse in the experiment. We plan to do more experiments to improve our results.

Figure 10 shows the measured energy spread as a function of time along the aforementioned beam. It is clear that there is higher energy spread at the beam head. The energy spread decreases from $\sim 12 \mathrm{eV}$ at the head to $\sim 2.2 \mathrm{eV}$ at the main beam, then goes up at the tail of the beam. The wiggle in the head of the beam may be caused by virtual cathode oscillation with a period of $\sim 5 \mathrm{~ns}$. The exact cause of the large energy spread at the head is not clear and more theoretical analysis is needed to understand it.

It is very interesting to compare the measured main beam energy spread with theoretical predictions. There are three main physical processes involved as the beam accelerates from the cathode to the measurement position: cooling due to acceleration, longitudinal-longitudinal effects, and the Boersch effect. These effects have been reviewed in Ref. [9]. Combining both the transverselongitudinal Boersch effect and the longitudinallongitudinal relaxation effect, the final beam energy spread can be expressed as

$$
\Delta \tilde{E}_{\| f}=\left[\frac{1}{\pi \varepsilon_{0}} q n^{1 / 3} q V_{0}+2 q V_{0} k_{B} T_{\|}\right]^{1 / 2}
$$

Here $\Delta \tilde{E}_{\| f}$ is the rms energy spread after acceleration and subsequent beam propagation, $q V_{0}$ is the beam energy, and $T_{\|}$is an increasing function of time or distance of beam propagation. Beam energy is in units of $\mathrm{eV}, n$ is beam density, and $q$ is the electron charge. The first term in the bracket corresponds to the longitudinallongitudinal effect, and the second term is the transverse-longitudinal (Boersch) effect. The longitudinal-longitudinal effect dominates in the initial accel-

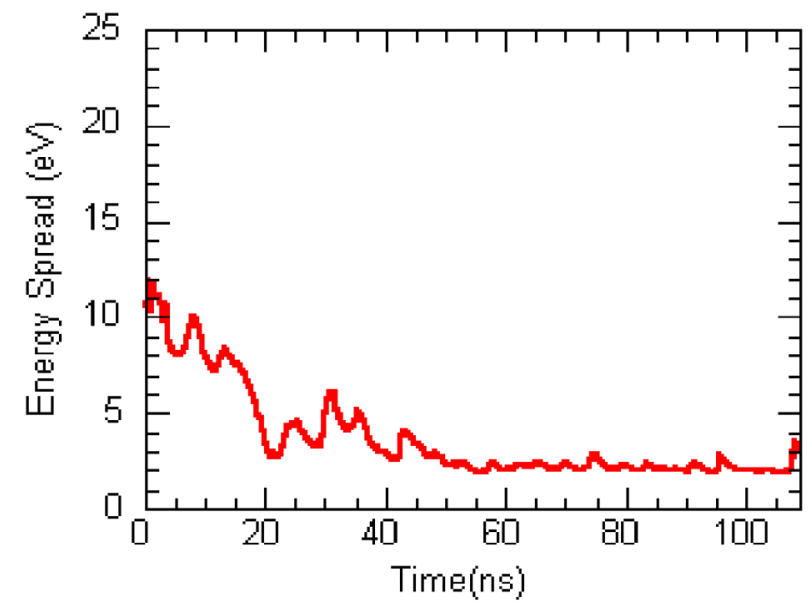

FIG. 10. (Color) Beam energy spread along beam pulse for a beam with energy of $5 \mathrm{keV}$ and current of $135 \mathrm{~mA}$. Average energy spread of the main beam is $2.2 \mathrm{eV}$. eration and propagation phase when there is a microfluctuation of the beam density distribution.

The energy spread due to the Boersch effect, on the other hand, increases monotonically until the longitudinal temperature reaches equilibrium. After a certain time of propagation, the Boersch effect will become the dominant source of the energy spread. According to the theoretical prediction, the rate of evolution of energy spread depends on the current density of the beam. The higher the beam density, the faster the beam energy spread increase in the longitudinal direction. In our experiment, the beam energy is $5 \mathrm{keV}$ and beam current is $135 \mathrm{~mA}$. The current density of the beam can be varied by changing the focusing strength of the solenoid. When we use a weak focusing strength, as shown with the black line in Fig. 5, the measured energy spread is $2.2 \mathrm{eV}$, which is very close to the theoretical prediction of $2.0 \mathrm{eV}$. On the other hand, when we use a strong focusing strength, as shown with the red line in Fig. 5, the current density gets higher, as does the beam energy spread. The measured energy spread increases to $2.5 \mathrm{eV}$, also very close to the theoretical prediction of $2.6 \mathrm{eV}$. We also measured the energy spread with beam energies of 3 and $4 \mathrm{keV}$. Beam current is $70 \mathrm{~mA}$ for the $3 \mathrm{keV}$ beam and $100 \mathrm{~mA}$ for the $4 \mathrm{keV}$ beam. Figure 11 shows both experimental and theoretical results. Triangles with solid line are the theoretical values for weak focusing. Diamonds with dotted line are the theoretical values for strong focusing. Circles are the measured energy spreads for weak focusing and squares are for strong focusing. Error bars added on the measured energy spread are determined by the resolution of the energy analyzer [10]. From this comparison, we can see the experimental results of the beam energy spread are in remarkably good agreement with the theoretical predictions. The maximum difference is only $0.2 \mathrm{eV}$ between the experimental results and theoretical predictions.

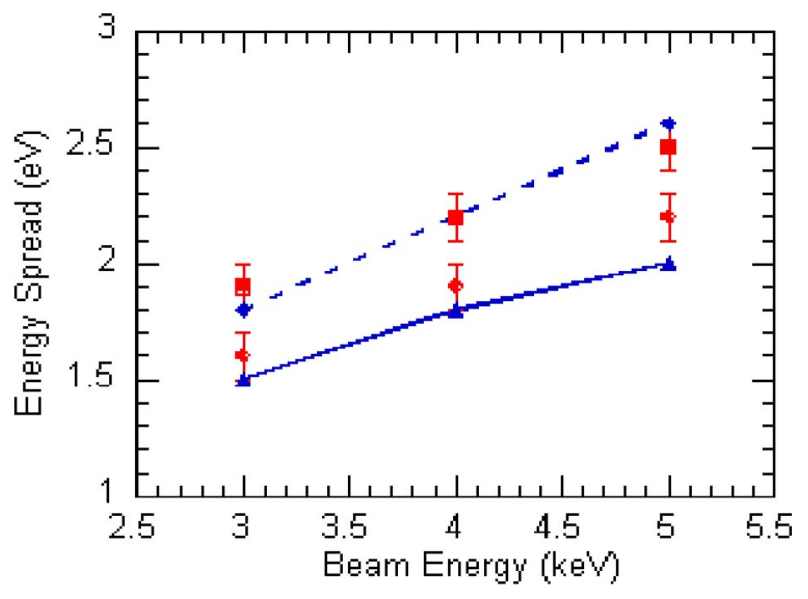

FIG. 11. (Color) Measured beam energy spreads compared with the theoretical predictions for different beam energies. 


\section{DERIVATION OF INPUT IMPEDANCE OF THE ELECTRON GUN}

Because of the high resolution of the energy analyzer, we are able to infer the input impedance of the gridded thermionic electron gun based on the beam mean energy measurement. Input impedance of the cathode is very important for electron gun performance. The triode electron gun consists of cathode, grid, and anode [15]. A basic electronic circuit of the triode electron gun is shown in Fig. 12. The gun electronics consist of a high voltage supply between the anode and the grid, a dc cathodegrid bias voltage $V_{B}(+30 \mathrm{~V})$ to suppress the beam during idle period, and a pulser circuit to provide a $\sim 100$ ns pulse signal delivered to the cathode by a transmission line with the characteristic impedance $Z_{0}$ of $50 \Omega$. The amplitude of the pulser source is $-2 V_{P}$. The source impedance $R_{s}$ of the pulser is $50 \Omega$. There is a $50 \Omega$ resistor $R$ at the output of the pulser circuit (between $\mathrm{A}$ and $\mathrm{B}$ ) to match the impedance of the rest circuits. The output voltage of the pulser (between A and B) is $-V_{P}$ $(-60 \mathrm{~V})$ in the match condition. All the electronics are located in a high voltage deck, which is isolated from ground and charged to $-V_{H}$. The cathode is biased by

(a)

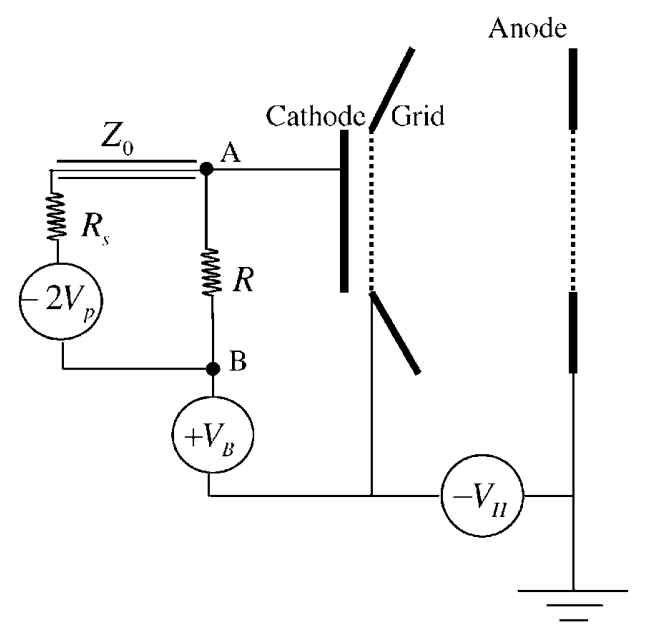

(b)

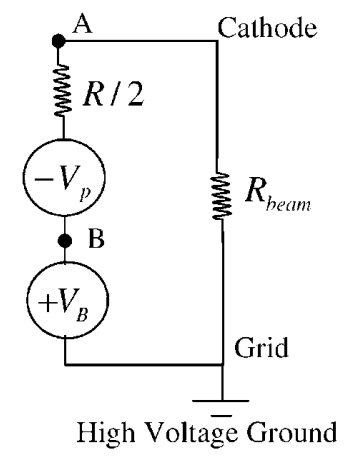

FIG. 12. (a) Basic electronic circuit of the triode electron gun. (b) Simplified equivalent circuit between the cathode and the grid of the gun. positive dc voltage relative to the grid to cut off the beam current. During emission, the pulse generator produces a negative pulse on the cathode to turn on the beam. The effect due to the magnetic field generated by the cathode heating current is minimized because the beam is emitted at the moment the ac heater current $(60 \mathrm{~Hz})$ crosses zero. A delay generator is employed to provide this synchronization.

In the experiment, when we change the cathode-grid bias voltage, both beam current and beam energy will change. Figure 13 shows the measured beam current versus the cathode-grid bias voltage when the grid high voltage $V_{H}$ is fixed at $5 \mathrm{kV}$. When the cathode-grid bias voltage is in the region of $0-30 \mathrm{~V}$, the beam current is space-charge limited and is almost a constant. When the bias voltage is larger than $30 \mathrm{~V}$, the beam current reduces dramatically. The beam energy is determined by two accelerating voltages: the high voltage $-V_{H}$ between the grid and the anode and the voltage $-V_{C G}$ between the cathode and the grid. We found that when we reduce the bias voltage from 30 to $20 \mathrm{~V}$, the mean energy measured by energy analyzer does not increase by $10 \mathrm{eV}$, but only by $5.8 \mathrm{eV}$. This, we believe, is caused by the impedance mismatch between the electronic circuit and the electron gun. Figure 12(b) is a simplified equivalent circuit between the cathode and the grid of the gun. The pulser circuit between the points $\mathrm{A}$ and $\mathrm{B}$ is equivalent to a pulser source with amplitude of $-V_{P}(-60 \mathrm{~V})$ in series with a resistance $R_{s}$ in parallel with $R$, which is $R / 2$. The equivalent impedance of the beam load between the cathode and grid is represented as $R_{\text {beam }}$, which only exists during beam emission.

As shown in Fig. 12(b), when triggered, the voltage between cathode and grid is

$$
-V_{C G}=\frac{R_{\text {beam }}}{R_{\text {beam }}+R / 2}\left(-V_{P}+V_{B}\right)
$$

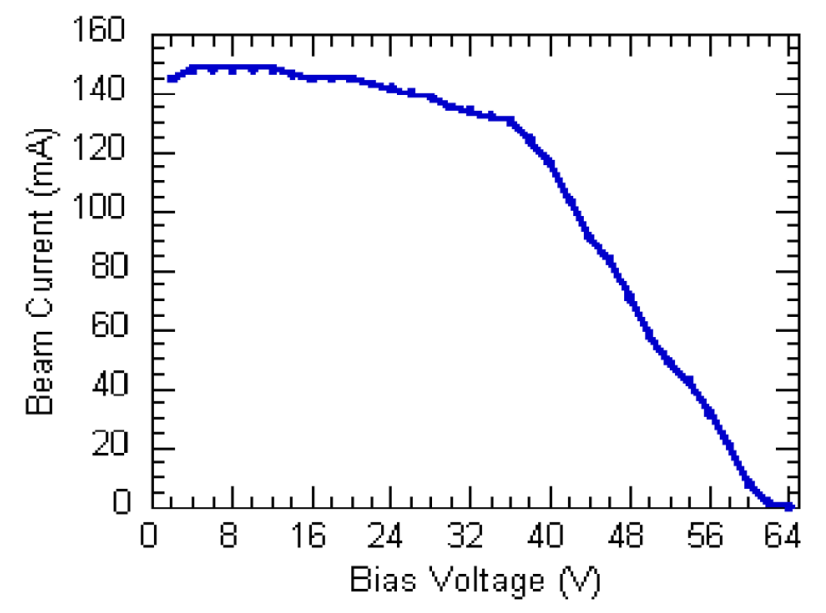

FIG. 13. (Color) Beam current between grid and anode versus bias voltage of the electron gun. 


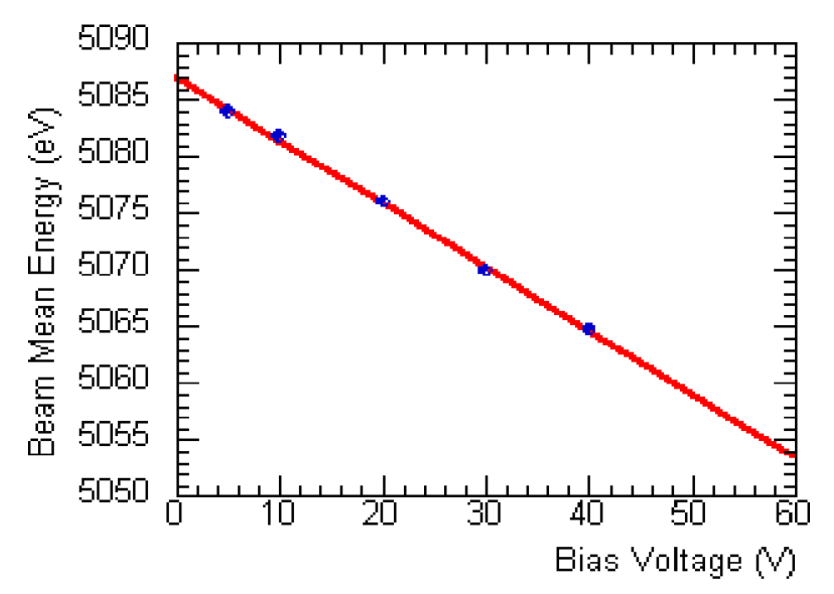

FIG. 14. (Color) Mean beam energy at different bias voltages (solid line is fitting line using experimental data shown as dots).

$-V_{C G}$ is the actual accelerating voltage between the cathode and the grid. After passing the grid, electrons are then accelerated by the high voltage $-V_{H}$, which does not change with the grid voltage. Figure 14 shows the mean energy of the beam at the different bias voltages. The solid line is a linear fitting to the experimental data, which are shown as dots. From the liner fit curve, the beam mean energy $E$ is related to the bias voltage by

$$
E=-0.56 V_{B}+5087 \text {. }
$$

Through the linear extrapolation, we can see that when the bias voltage is zero, the beam energy is at $5087 \mathrm{eV}$, and when the bias voltage is equal to pulse voltage of $60 \mathrm{~V}$, the mean beam energy is at $5053 \mathrm{eV}$. From the information of the mean beam energy at different bias voltages, we can calculate the net voltage between the cathode and the grid as follows. When the bias voltage $V_{B}$ equals the pulse voltage $V_{P}$, we know the cathode-grid voltage $V_{C G}$ is zero according to Eq. (1). When the bias voltage is at other values, the cathode-grid voltage can be calculated as

$$
V_{C G}\left(V_{B}\right)=E\left(V_{B}\right)-E\left(V_{B}=60 \mathrm{~V}\right) .
$$

The results of the calculation are shown in Fig. 15. We can see when bias voltage changes from 0 to $40 \mathrm{~V}$, the amplitude of the voltage between the cathode and the grid reduces from 34 to $11 \mathrm{~V}$.

From Eq. (1) we can get the beam impedance between the cathode and the grid,

$$
Z_{\text {beam }}=\frac{V_{C G}}{2\left(V_{P}-V_{B}-V_{C G}\right)} R .
$$

Here, $R$ is the $50 \Omega$ matching resistor.

In the experiments, $V_{P}$ is fixed at $60 \mathrm{~V}, V_{C G}$ and $V_{B}$ are both known from Fig. 15. So when we change the grid bias voltage $V_{B}$ in the gun's saturation region, we can calculate the beam impedance at different voltages, the result of which is shown by the dots in Fig. 16. It is interesting that the beam impedance is about $30 \Omega$ and

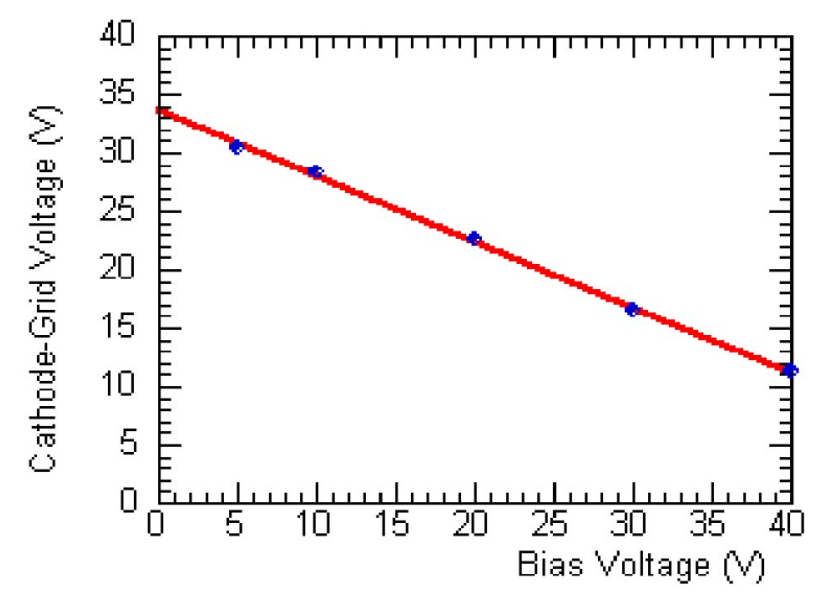

FIG. 15. (Color) Voltage amplitude between the cathode and the grid at the different bias voltages (solid line is fitting line using experimental data shown as dots).

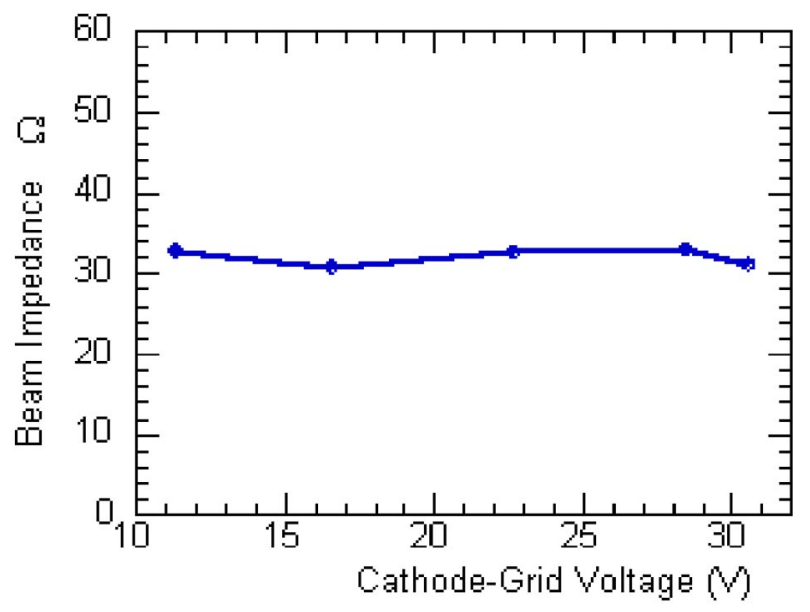

FIG. 16. (Color) Beam impedance versus the voltage between the cathode and the grid.

therefore the $V-I$ characteristic between the cathode and the grid is linear in the gun's saturation region. Triode gun behavior working in the saturation region is very complicated and there are currently no accurate mathematic models to describe its current and voltage relation in its saturation region. The energy analyzer provides one indirect way to measure the beam impedance on the cathode.

\section{CONCLUSION}

Beam energy spread and mean energy have been measured at the exit of a gridded electron gun using a highresolution cylindrical retarding field energy analyzer. The measured beam energy spreads are in remarkably good agreement with the intrinsic limits set by the effects of nonadiabatic acceleration in the electron gun and that of Coulomb collisions, as predicted by theory. The absolute mean energy is believed to have a dc offset from the real 
beam energy due to the field leakage at the retarding mesh. The offset is about $1 \%$ of the beam energy. However, the measurement of the relative beam energy and the energy spread has very high resolution, better than $0.2 \mathrm{eV}$. By accurately measuring the beam energy change with the grid bias voltage, we are able to calculate the input impedance between the cathode and grid due to beam loading.

\section{ACKNOWLEDGMENTS}

The work is supported by DOE Grants No. DEFG0292ER54178 and No. DEFG0294ER40855. Also, the authors thank Dr. Mark T. Walter for mechanical support and Mr. John Harris for useful suggestions.

[1] M. Reiser, Theory and Design of Charged Particle Beams (Wiley, New York, 1994).

[2] S. Ichimaru and M. N. Rosenbluth, Phys. Fluids 13, 2778 (1970).

[3] T. M. O'Neil and P.G. Hjorth, Phys. Fluids 28, 3241 (1985).

[4] H. Boersch, Z. Phys. 139, 115 (1954).

[5] A.W. Hyatt, C.F. Driscoll, and J.H. Malmberg, Phys. Rev. Lett. 59, 2975 (1987).

[6] A.V. Aleksandrov, N.S. Dikansky, N.Cl. Kot, V. I. Kudelainen, V. A. Lebedev, P.V. Logachov,
R. Calabrese, V. Guidi, G. Ciullo, G. Lamanna, and L. Tecchio, Phys. Rev. A 46, 6628 (1992).

[7] P. G. O'Shea, M. Reiser, R. A. Kishek, S. Bernal, H. Li, M. Pruessner, V. Yun, Y. Cui, W. Zhang, Y. Zou, T. Godlove, D. Kehne, P. Haldemann, and I. Haber, Nucl. Instrum. Methods Phys. Res., Sect. A 464, 646 (2001).

[8] H. Suk, Ph.D. thesis, University of Maryland, 1996.

[9] Y. Zou, Y. Cui, V. Yun, A. Valfells, R. A. Kishek, S. Bernal, I. Haber, M. Reiser, P. G. O'Shea, and J. G. Wang, Phys. Rev. ST Accel. Beams 5, 072801 (2002).

[10] Y. Cui, Y. Zou, A. Valfells, M. Reiser, M. Walter, I. Haber, R. A. Kishek, S. Bernal, and P.G. O'Shea (to be published).

[11] Y. Zou, Y. Cui, I. Haber, M. Reiser and P. G. O'Shea, Phys. Rev. ST Accel. Beams 6, 112801 (2003).

[12] A. Faltens, E. P. Lee, and S. S. Rosenblum, J. Appl. Phys. 61, 5219 (1987).

[13] J. G. Wang, H. Suk, D. X. Wang, and M. Reiser, Phys. Rev. Lett. 72, 2029 (1994).

[14] J. Harris, A. Valfells, B. Beaudoin, S. Bernal, A. Diep, I. Haber, Y. Huo, B. Quinn, M. Reiser, M. Walter, and P. G. O'Shea, in Proceedings of the 2003 Particle Accelerator Conference, Portland, OR, 2003 (IEEE, New York, 2003), pp. 2312-2314.

[15] J. G. Wang, E. Boggasch, P. Haldemann, D. Kehne, M. Reiser, T. Shea, and D.X. Wang, IEEE Trans. Electron Devices 37, 2622 (1990). 